\title{
Wet Chemical Synthesis of Cadmium Sulphide Nanoparticles and its Characterization +
}

\author{
S. RAMESH and V. NARAYANAN* \\ Department of Inorganic Chemistry, University of Madras, \\ Guindy Campus, Chennai-600025, India \\ vnnara@yahoo.co.in
}

Received 22 January 2013 / Accepted 15 February 2013

\begin{abstract}
Cadmium sulphide (CdS) nanoparticles were successfully prepared by wet chemical method using cadmium acetate and sodium sulphide as the cadmium and sulphide sources along with sodium citrate as the capping agent. The synthesized CdS nanoparticles were characterized by XRD, SEM in order to confirm the phase purity and morphology of the sample. CdS nanoparticles exhibit cubic structure, which was revealed by the XRD analysis.
\end{abstract}

Keywords: Wet Chemical Synthesis, Cadmium sulphide nanoparticles, XRD analysis

\section{Introduction}

One-dimensional (1D) nanostructures, such as nanowires, nanorods, nanofibres and nanotubes, are very interesting materials because they have anisotropic geometries and unique physical properties $^{1,2}$. The 1D II-VI semiconductor nanocrystals are well studied and have been achieved in recent years. CdS is one of the most important materials which has a direct band gap of $2.5 \mathrm{eV}$ for the bulk hexagonal wurtzite structure, and $3.53 \mathrm{eV}$ for bulk cubic zinc blende structur ${ }^{\mathrm{e} 3,4}$. CdS has a potential application in light emitting diodes, solar cells, optoelectronics, and photocatalysts, ${ }^{5,6}$. It has been synthesised by a variety of methods, including solvothermal reaction $^{5}$, thermal evaporation ${ }^{7}$ and microwave irradiation ${ }^{6}$. The synthesis of colloidal inorganic nanocrystals especially with respect to the control of their shape, however, is under developed and still complicated. Since novel properties of nanomaterials depend on their size and shape, a new direction for synthetic methods and an understanding of the mechanisms by which the size and shape of the nanocrystals can be easily varied are key issues in nanochemistry ${ }^{8,9}$. The formation of high-quality semiconductor nanocrystal, with well-controlled size and shape as well as well-confined surface passivation, can be achieved by controlling the thermodynamics and kinetics during the nucleation and growth of nanocrystals. $(\mathrm{NCs})^{8,9}$. Various methods of synthesis of nanoparticles with a series of anisotropic structures like nanorods, bipods, nanocubes and nanowires have been well established by various groups ${ }^{10,11}$. In the present work, we have synthesized CdS by wet chemical synthesis using sodium citrate as capping agent.

$†$ Presented to the National Conference on Chemistry Solutions at SRM University, India 


\section{Experimental}

Cadmium chloride, sodium sulphide, sodium citrate were purchased from Qualigens. Double distilled water was used as the solvent throughout the experiment.

\section{Characterization methods}

The crystal structure of CdS nanoparticles was analyzed by a Rich Siefert 3000 diffractometer with $\mathrm{Cu}-\mathrm{K} \alpha_{1}$ radiation $(\lambda=1.5406 \AA)$. The morphology of the materials was analyzed by SEM HITACHI SU6600 scanning electron microscopy respectively.

\section{Wet synthesis of CdS nanoparticles}

$\mathrm{CdS}$ was prepared by stirring $1 \mathrm{mM}$ of cadmium chloride with $5 \mathrm{mM}$ sodium citrate along with addition of $1 \mathrm{mM}$ of sodium sulphide. The precipitate was washed with double distilled water twice and dried at $60{ }^{\circ} \mathrm{C}$ in air.

\section{Results and Discussion}

\section{Structure and morphology}

XRD patterns of the CdS nanoparticles is shown in Figure 1, which indicates the CdS has cubic phase structure. The peak positions $(2 \theta=26,45.6)$ and relative intensities obtained for the CdS match with the JCPDS card No: 21-829 file, identifying it as CdS with a cubic structure and cell constant $\mathrm{a}=5.45 \AA$. There was no characteristic peaks of impurity were observed. The average grain size of $\mathrm{CdS}$ is determined using Scherrer relation and it was found to be around $15 \mathrm{~nm}$.

The SEM micrograph of the CdS calcined is shown in Figure 2. It can be seen that the particles adopt irregular morphology with different sized particle. From the image it is clear that the particles were highly agglomerated in nature. This might be due to the fact that the agglomeration may be induced during the crystal growth itself because of the small size regime which is evident from the XRD analysis.

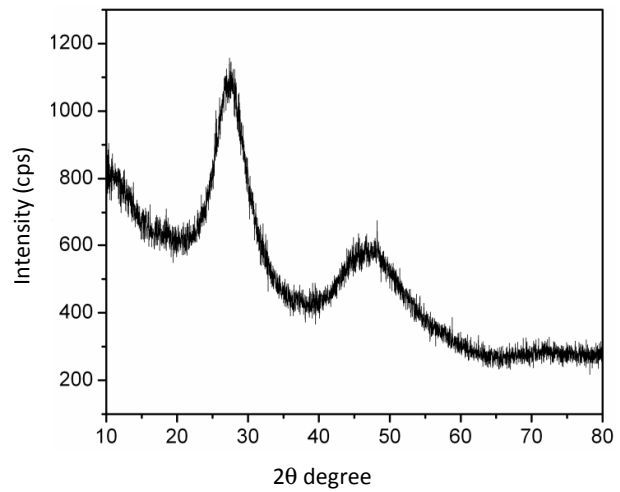

Figure 1. XRD pattern of the CdS nanoparticles.

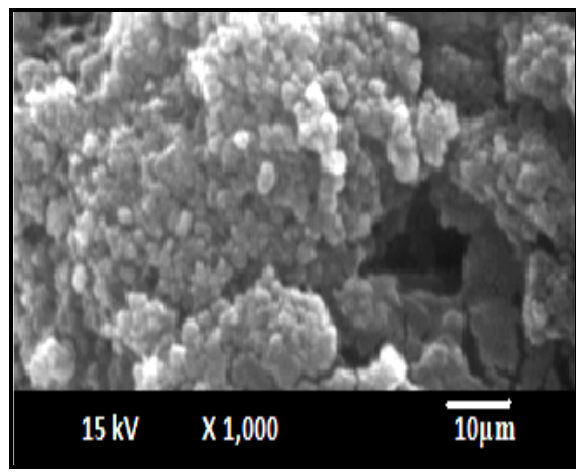

Figure 2. SEM image of the CdS nanoparticles.

\section{Conclusion}

CdS nanoparticles were synthesised by simple one step wet chemical synthesis. The synthesised CdS was characterised by XRD and SEM. From XRD analysis, it is understood that the nanoparticles were small in nature with an average grain size of $15 \mathrm{~nm}$. SEM analysis showed the particles were highly agglomerated with particle size of 10-25 nm. 


\section{References}

1. Fan H M. Fan X F, Ni Z H, Shen Z X, Feng Y P and Zou B S, J Phys Chem C, 2008, 112, 1865-1870.

2. Lee S M, Cho S N and Cheon J, Adv Mater., 2003, 15, 441-444.

3. Zeiri L, Patla I, Acharya S, Golan Y and Efrima S, J Phys Chem C, 2007, 111, 11843 11848.

4. Cao H, Wang G, Zhang S, Zhang X and Rabinovich D, Inorg Chem., 2006, 45, 51035108.

5. Thongtem T, Phuruangrat A and Thongtem S, Mater Lett., 2007, 61, 3235-3238.

6. Thongtem T, Phuruangrat A and Thongtem S, J Phys Chem Solids., 2008, 69, 13461349.

7. Pan A, Liu R, Yang Q, Zhu Y, Yang G, Zou B and Chen K, J Phys Chem B, 2005, 109(51), 24268-24272.

8. Murray C B, Norris D J and Bawendi M G, J Am Chem Soc., 1993, 115, 8706-8715.

9. Martin C R, Science, 1994, 266, 1961-1966.

10. Wang J, Gudiksen M S, Duan X, Cui Y and Lieber C M, Science, 2001, 293, 1455-1457.

11. Xia Y, Yang P, Sun Y, Wu Y, Mayers B, Gates B, Yin Y, Kim F and Yan H, Adv Mater., 2003, 15, 353-389. 